\title{
Gnrh mRNA expression in the brain of cooperatively breeding female Damaraland mole-rats
}

\author{
Cornelia Voigt and Nigel C Bennett \\ Department of Zoology and Entomology, University of Pretoria, Pretoria, South Africa \\ Correspondence should be addressed to C Voigt; Email: cvoigt@zoology.up.ac.za
}

\begin{abstract}
The Damaraland mole-rat (Fukomys damarensis) is a eusocial, subterranean rodent, in which breeding is limited to a single reproductive pair within each colony. Non-reproductive females, while in the confines of the colony, exhibit socially induced infertility. Anovulation is thought to be caused by a disruption in the normal gonadotropin-releasing hormone (GNRH) secretion from the hypothalamus. To assess whether social suppression is associated with altered Gnrh mRNA expression in the brain, we investigated the distribution and gene expression levels by means of in situ hybridization in female breeders and non-breeders from field captured colonies of the Damaraland mole-rat. We found expression of Gnrh mRNA as a loose network in several forebrain areas of female Damaraland mole-rats with the majority of labelling in the preoptic and anterior hypothalamus. The distribution matched previous findings using immunocytochemistry in this and other social mole-rat species. Quantification of the hybridisation signal revealed no difference between breeding and non-breeding females in the average optical density of the hybridization signal and the size of the total area covered by Gnrh mRNA. However, analysis along the rostro-caudal axis revealed significantly elevated Gnrh mRNA expression in the rostral preoptic region of breeders compared to non-breeders, whereas the latter had increased Gnrh mRNA expression at the caudal level of the anterior hypothalamus. This study indicates that social suppression affects the expression of Gnrh mRNA in female Damaraland mole-rats. Furthermore, differential regulation occurs within different neuron subpopulations. Reproduction (2017) 153 453-460
\end{abstract}

\section{Introduction}

In cooperatively breeding birds and mammals, reproductive skew is maintained by reproductive suppression of subordinate group members (Keller \& Reeve 1994). Socially induced suppression of ovulation represents one such mechanism in female mammals and is best known from callitrichid primates such as the common marmoset (Callithrix jacchus, Abbott 1984) and eusocial mole-rats (Faulkes et al. 1990, Molteno \& Bennett 2000). In these species, anovulation of subordinates appears to occur due to an insufficient release of luteinizing hormone (LH) from the anterior pituitary.

African mole-rats (Bathyergidae) exhibit a wide range of social organisation, from strictly solitary to highly social species, and thus, differ in the degree of reproductive skew (Faulkes \& Bennett 2001). Previous studies investigating the GNRH system by means of immunocytochemistry (ICC) have revealed inconsistent results. Among the social species, where breeding is restricted to a single female (queen) within the group, reproductive status had either no effect on the morphology and immunoreactivity of GNRH neurons (common mole-rat, Du Toit et al. 2006; naked molerat, Zhou et al. 2013) or non-breeders differed from breeders by showing elevated immunoreactivity in the median eminence and/or higher brain GNRH content (Damaraland mole-rat, Molteno et al. 2004; Highveld mole-rat, Du Toit et al. 2006; Natal molerat, Oosthuizen et al. 2008). The latter would indicate a reduced rate of GNRH release into the hypophyseal portal blood system, resulting in inadequate activation of the anterior pituitary of such suppressed females.

Damaraland mole-rats (Fukomys damarensis) with an average group size of 11 individuals represent an extreme example of socially induced infertility, in that reproduction is completely blocked in subordinate female group members. Such females show no signs of sexual behaviour, have significantly lower urinary oestradiol and progesterone levels and exhibit interrupted follicular development compared to the dominant breeding female of the group (Bennett 1994, Bennett et al. 1994). Therefore, the blockade of reproduction results from an inhibition of ovulation, which appears to be caused by inadequate secretion of luteinizing hormone (LH) from the pituitary, which in 
turn might be brought about by a disruption of the normal GNRH secretion from the hypothalamus (Molteno et al. 2004). However, females removed from the presence of the queen start to ovulate spontaneously (Molteno \& Bennett 2000, Snyman et al. 2006). Furthermore, we have shown recently that reproductive status and social suppression affect the neuroendocrine phenotype of female Damaraland mole-rats with subordinates having reduced expression of androgen and estrogen receptor $\alpha$ genes in several preoptic-hypothalamic brain regions when compared to breeders (Voigt et al. 2014).

The question arises as to whether the block to ovulation lies at the level of the hypothalamus, i.e. the GNRH neurons or at the level of the anterior pituitary, i.e. the GNRH receptors or both. Using ICC and radioimmunoassay (RIA), Molteno and coworkers (2004) investigated the distribution and morphology of GNRH-immunoreactive structures and the GNRH content in the brain of female breeders and non-breeders and of females removed from suppression. Although reproductive status did not affect the size and the total number of GNRH cells, suppressed females had a larger amount of GNRH in the median eminence and in the proximal pituitary stalk, suggesting that their release from the nerve terminals is inhibited (Molteno et al. 2004). These data would be indicative of suppression within the hypothalamus. On the other hand, subordinate females secrete significantly less $\mathrm{LH}$ in response to a single dose of exogenous GNRH than breeders (Bennett et al. 1993), which suggests that the anterior pituitary of such females is desensitized. GNRH receptors are positively autoregulated by physiological concentrations of GNRH (Clayton 1982); thus, a lack of GNRH may lead to their downregulation. There is evidence from several studies in rats that GNRH biosynthesis is linked to GNRH and LH release (Wise et al. 1981, Zoeller \& Young 1988, Petersen et al. 1996, Spratt \& Herbison 1997, Jimenez-Linan \& Rubin 2001). For example, hypothalamic Gnrh mRNA levels were found to increase at proestrus in female rats prior to the preovulatory LH surge (Gore \& Roberts 1995, Petersen et al. 1996, Jimenez-Linan \& Rubin 2001). Therefore, the present study aims at investigating whether levels of Gnrh mRNA are altered between reproductively active and suppressed females, which may provide an indication for differential transcriptional regulation of the GNRH gene. Using in situ hybridization, we analysed the distribution, optical density and area covered by Gnrh mRNA in the forebrain of breeding females and of non-breeding females.

\section{Materials and methods}

\section{Animals}

In the current study, adult female Damaraland mole-rats (Fukomys damarensis) were used. Data were obtained from eight reproductive females (breeder, syn. queen) and eight non-reproductive females (non-breeder). The non-breeders derived from colonies captured between April and June 2013 near the village of Black Rock, Northern Cape, South Africa $\left(27^{\circ} 7^{\prime} \mathrm{S}, 22^{\circ} 50^{\prime} \mathrm{E}\right)$ with Hickman live-traps under permission from Northern Cape Nature Conservation authorities. Prior to sacrifice, animals were housed for a maximum period of 10 weeks at the University of Pretoria under 12 L:12 D cycle at $25^{\circ}$ in plastic containers $(1.0 \mathrm{~m} \times 0.5 \mathrm{~m} \times 0.5 \mathrm{~m})$ containing wood shavings, and they were fed on sweet potato, gem squash and apples. The breeders were captured in December 2012 as subordinates and kept in the laboratory paired with a male, respectively, for 7 months before sacrifice. Reproductive activity was confirmed for all pairs by the observation of copulations. Four females were pregnant at the time of sacrifice. The reproductive status of the females was confirmed post mortem by examination of the reproductive tract. At the time of brain collection, body mass of all animals was recorded to the nearest gram. All experimental procedures were approved by the University of Pretoria Animal Ethics Committee (EC003-12).

\section{Brain histology}

Mole-rats were killed by decapitation, brains were dissected out of the skull, immediately frozen on dry ice and stored at $-80^{\circ} \mathrm{C}$ until used. Before sectioning, brain mass was recorded to the nearest milligram. Frozen brains were cut on a cryostat into $30 \mu \mathrm{m}$ coronal sections. The plane of the sections was adjusted to match as closely as possible to the plane of the rat brain atlas (Paxinos \& Watson 2007). Sections were mounted onto Superfrost Plus slides (Menzel-Gläser, Braunschweig, Germany) in four different series, so that one series of slides contained a section every $120 \mu \mathrm{m}$. In situ hybridization was carried out on every fourth section for the localization of Gnrh mRNA.

\section{Cloning of cDNA probe}

Based on sequence information available from other species, PCR was used to amplify a fragment of the Gnrh 1 gene from the Damaraland mole-rat. Total RNA was extracted from mole-rat hypothalamus using the RNeasy Mini Kit (Qiagen $\mathrm{GmbH}$ ). The synthesis of first-strand cDNA was done with SUPERSCRIPT III Reverse Transcriptase (Invitrogen) and random primers. The resulting RNA-DNA hybrids were subsequently used in PCR to generate pieces of the appropriate gene. The forward primer was 5'-ACAATACTCAACACAAGCTTGA-3' and the reverse primer was 5'-ATCTATAAACTGTGCGGCTCTCA-3'. PCR was carried out for 40 cycles using the following parameters: $94^{\circ} \mathrm{C}$ for $1 \mathrm{~min}, 58^{\circ} \mathrm{C}$ for $45 \mathrm{~s}, 72^{\circ} \mathrm{C}$ for $1 \mathrm{~min}$. The amplified fragment was purified and cloned into the pCRII TOPO vector using the TOPO-TA cloning kit (Invitrogen). Resultant clones were sequenced to verify the authenticity and fidelity of the amplification. The cloned Gnrh 1 sequence (GenBank: KX555603) is $421 \mathrm{bp}$ in length and encompasses the entire coding region ( $279 \mathrm{bp}$ ) plus $51 \mathrm{bp}$ of the $5^{\prime}$ untranslated region and 91 bp of the $3^{\prime}$ untranslated region. The coding region shows 

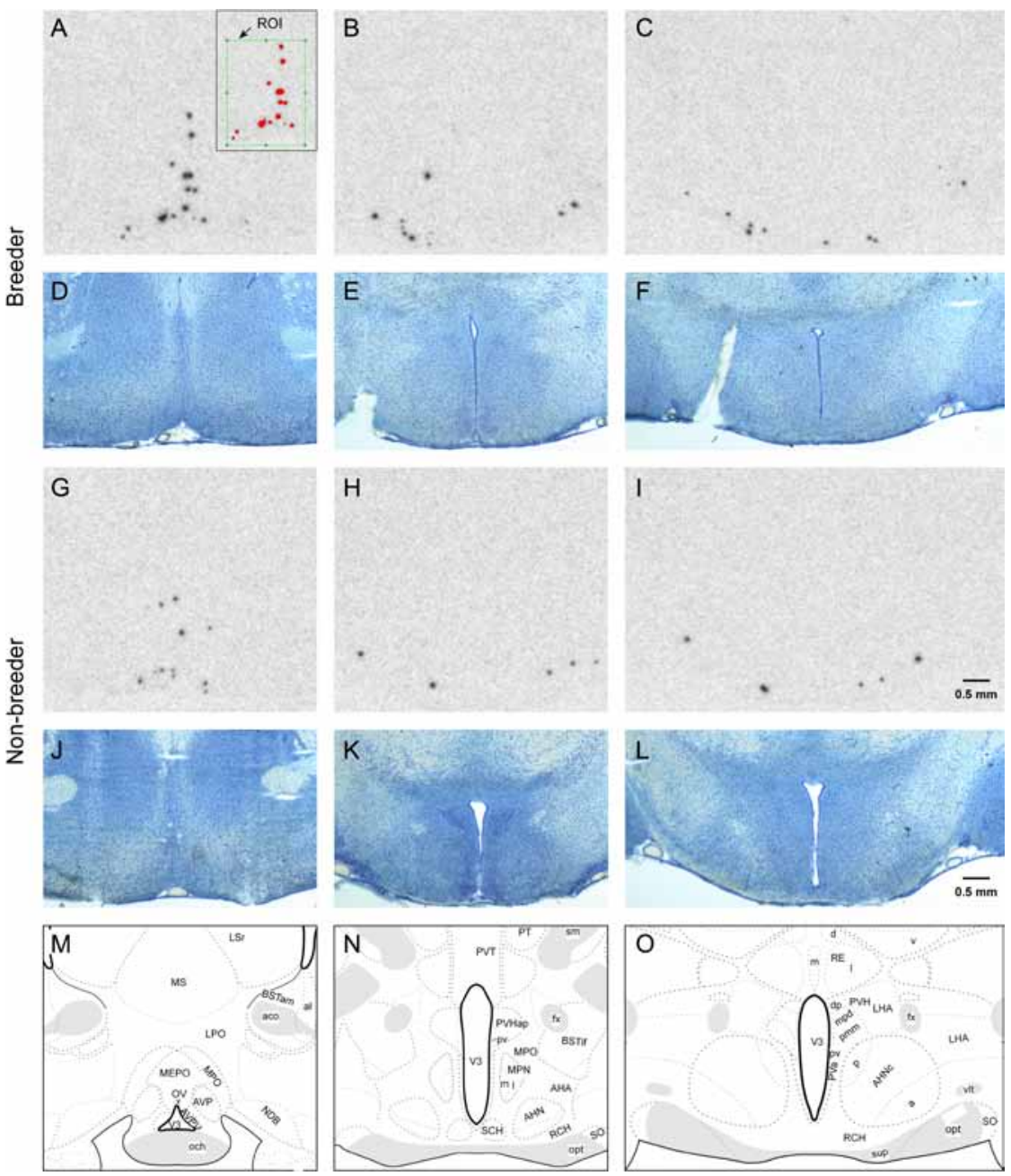

Figure 1 Brightfield photomicrographs of autoradiograms, corresponding Nissl-stained sections and schematic drawings of coronal sections from the brain of a female breeder and non-breeder Damaraland mole-rat illustrating the distribution of Gnrh mRNA (dark spots) visualized by in situ hybridization. (A, D, G, J and M) Sections from the anterior preoptic region at the level of the MS/NDB (AC+3). (B, E, H, K and N) Sections at the level of the suprachiasmatic nucleus (AC-8). (C, F, I, L and O) Sections at the level of retrochiasmatic area, rostral to the arcuate nucleus (AC-11). aco, anterior commissure, olfactory limb; $\mathrm{AHA}$, anterior hypothalamic area; $\mathrm{AHNa}, \mathrm{c}, \mathrm{p}$, anterior hypothalamic nucleus anterior, central, posterior part; AVP, anteroventral preoptic nucleus; AVPV, anteroventral periventricular nucleus hypothalamus; BSTal, am, bed nuclei stria terminalis, anterior division, anterolateral area, anteromedial area; BSTif, bed nuclei stria terminalis, posterior division, interfascicular nucleus; fx, fornix; LHA, lateral hypothalamic area; LPO, lateral preoptic area; LSr, lateral septal nucleus, rostral part; MEPO, median preoptic nucleus; MPNm, I, medial preoptic nucleus, medial, lateral part; MPO, medial preoptic area; MS, medial septal nucleus; NDB, diagonal band nucleus; och, optic chiasm; opt, optic tract; OV, vascular organ of the lamina terminalis; PT, paratenial nucleus, PVa, periventricular hypothalamic nucleus, anterior part; PVHap, dp, mpd, pmm, paraventricular hypothalamic nucleus (anterior parvicellular, dorsal parvicellular, medial parvicellular, posterior magnocellular part); PVT, paraventricular thalamic nucleus; RE, nucleus reuniens; RCH, retrochiasmatic area; $\mathrm{SCH}$, suprachiasmatic nucleus; sm, stria medullaris; SO, supraoptic nucleus; sup, supraoptic commissures; V3, third ventricle; vlt, ventrolateral hypothalamic tract. Adapted from Swanson (2004).

$97 \%$ homology with a previously cloned sequence $(227 \mathrm{bp})$ from Highveld mole rats (Cryptomys hottentotus pretoriae, (GenBank: AJ966357.1) (Kalamatianos et al. 2005), 77\% homology with rat Gnrh1 (GenBank: NM_012767) and 86\% homology with guinea pig Gnrh 1 (GenBank: NM_001172956). The GNRH peptide of the latter species is unique in that it differs from the form found in all other mammals by two amino acid substitutions. Moreover, two polyadenylation sites are present in the precursor mRNA, resulting in two transcripts (Jimenez-Linan et al. 1997). Despite the high homology with guinea pig Gnrh1, the sequences isolated from the two mole-rat species represent the 'mammalian' GNRH.

\section{In situ hybridization}

The expression of GNRH in brain sections was detected with antisense RNA probes labelled with ${ }^{35} \mathrm{~S}$-CTP. Labelling of the probes with ${ }^{35} \mathrm{~S}-\mathrm{CTP}(1250 \mathrm{Ci} / \mathrm{mmol}$; Perkin Elmer) was performed using the Riboprobe System (Promega). Our in situ hybridization procedure followed a previously 


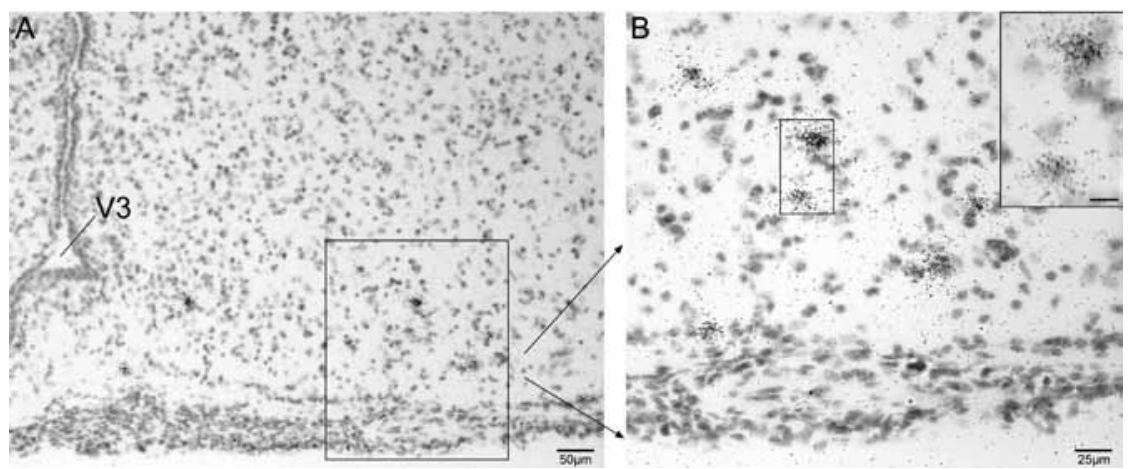

Figure 2 Representative emulsion-coated sections from in situ hybridization illustrating Gnrh mRNA expression at the level of the anterior commissure. Cell nuclei are counterstained with cresyl violet and Gnrh mRNA labelling is represented by clusters of black silver grains. (A) The boxed region is shown in B at a higher magnification. (B) Clusters of silver grains located over cell bodies. Inset shows two clusters at $100 \times$ magnification. $\mathrm{V} 3$, third ventricle. Scale bars $=50 \mu \mathrm{m}$ in $\mathrm{A} ; 25 \mu \mathrm{m}$ in $\mathrm{B} ; 10 \mu \mathrm{m}$ for the inset in B. published protocol (Whitfield et al. 1990) with modifications as described elsewhere (Gahr \& Metzdorf 1997). For signal detection, sections were exposed to autoradiographic film (Kodak Biomax MR, Rochester, NY, USA) for 3 weeks. Brain sections from both groups of females were run through the entire procedure at the same time and placed on each autoradiographic film to avoid any possible effect of small differences in procedures on the observed group differences. Control sections processed with the sense probe were obtained from two female mole-rats and were labelled by the same procedure as described previously. Autoradiograms from these sections showed no signal. These control data will therefore not be discussed below. In addition, 5 sections from one female mole-rat were processed with NTB photographic emulsion (Carestream Health, Stuttgart, Germany) for 7 days. Sections were developed with Kodak Dektol Developer and Kodak Fixer and counterstained with cresyl violet.

\section{Data analysis}

Images from autoradiograms were scanned with an Epson Perfection V750 Pro scanner connected to a PC running the image analysis software Image $1.50 u(\mathrm{NIH}$; see http://rsb. info.nih.gov/ij/). To obtain the output of the measurements in optical density (OD) the system was calibrated using a calibrated optical density step tablet (T2115CC; Stouffer Industries, Inc., Mishawaka, IN, USA) and a calibration curve was fitted with the Rodbard function of ImageJ $\left(y=d+(a-d) /\left(1+(x / c)^{\wedge} b\right)\right)$. This calibration was applied to all images, and it extended beyond the darkest spot to be measured in the autoradiograms so that the signals that were measured did never reach saturation. Anatomical landmarks were obtained by light microscopic examination of the counterstained sections that were used for the autoradiograms and additionally, of Nissl-stained similar sections from other individuals that had not been processed with in situ hybridization. Sections of all animals were realigned according to the anterior commissure (AC) at the level where it reaches its largest extension.

To quantify the level of Gnrh mRNA expression, the image was converted to an 8-bit grayscale image. A thresholding approach was used to measure the optical density and the area of the hybridization signal. For each section, to obtain a threshold value that separates the background from the specific labelling, I chose a rectangular area $\left(1 \mathrm{~mm}^{2}\right)$ immediately ventral to the brain section of interest. It must be noted that outside the areas expressing GNRH the rest of the brain was always at an optical density close to the film background. Using the threshold function in Imagel, a threshold value was set manually, which resulted in the thresholded region being displayed in colour (Fig. 1A). The selection tool was used to mark the region of interest (ROI). The mean OD and area size of the colour-marked region within the ROI was obtained using the measurement function of the program applying the option 'limit to threshold'. The film background OD was obtained from a $1 \mathrm{~mm}^{2}$ rectangular area ventral to the brain section. The final OD measurement was the difference between the film background OD value and the OD value of the ROI. Quantification of the mRNA expression level was done in adjacent sections along the rostro-caudal axis within the preoptic and the anterior hypothalamus. The measurements comprised a region, which ranged from four sections rostral to the anterior commissure $(A C+4)$ up to twelve sections caudal of the anterior commissure (AC-12).

\section{Statistical analysis}

Statistical analyses were carried out using JMP software. Data are presented as means \pm S.E.M. Morphological differences and differences in the average optical density and total area covered by Gnrh mRNA between groups were analysed with t-tests. For analysis of GNRH gene expression along the rostrocaudal axis, a REML-model was employed with reproductive status and brain region as fixed factors and animal ID as random factor. Post hoc analysis was performed using the 'test slices' comparison in JMP. All tests were two-tailed, and the significance level was fixed at $P<0.05$.

\section{Results \\ Morphological measurements}

The two groups of females differed significantly in body mass with breeders $(98.3 \pm 3.7 \mathrm{~g})$ being heavier than non-breeders $(83.9 \pm 3.9 \mathrm{~g}, t=2.66, d f=14, P=0.018)$. No significant group differences were found in brain mass $(t=0.95, d f=14, P=0.36)$.

\section{Distribution of Gnrh mRNA}

The neuroanatomical distribution of Gnrh mRNA matched largely the distribution of GNRH-ir cell 


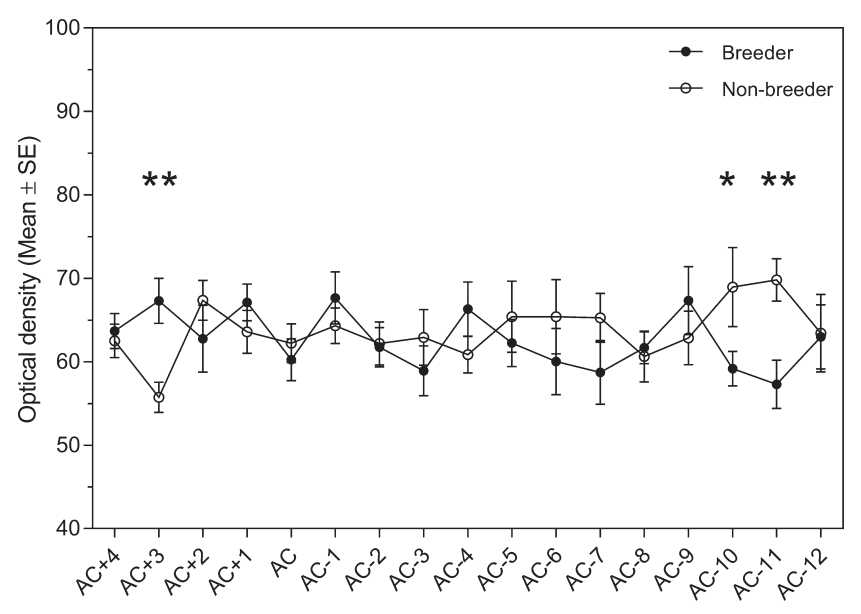

Figure 3 Average optical density of the hybridisation signal for Gnrh mRNA along the rostro-caudal axis of the preoptic and anterior hypothalamus in female breeders and non-breeders $(* * P<0.01$, $* P<0.05)$. AC, anterior commissure.

bodies reported previously in the same species and in other mole-rats (Molteno et al. 2004, Du Toit et al. 2006, Oosthuizen et al. 2008, Zhou et al. 2013). Gnrh mRNA expression occurred as a loose network throughout the rostro-caudal extent of the forebrain (Figs 1 and 2). Labelling was most pronounced in the preoptic and in the anterior hypothalamus. In the anterior preoptic region, dorsally, labelling was close to the midline corresponding to the median preoptic nucleus and the medial septal nucleus. Ventrally it occurred more laterally, corresponding to the anteroventral and medial preoptic nucleus and the diagonal band nucleus (Fig. 1A, G and M). In the anterior hypothalamus, labelling occurred laterally and extended in rostro-caudal direction within the lateral retrochiasmatic area and the anterior hypothalamic area and in close proximity to and occasionally within the supraoptic nucleus (Fig. 1B, C, H, I, N and $\mathrm{O}$ ). Occasionally, labelling was found in the suprachiasmatic nucleus. In the tuberal hypothalamus, labelling occurred along the retrochiasmatic part of the supraoptic nucleus. No differences were seen in the distribution of the Gnrh mRNA between breeder and non-breeder females.

\section{Gnrh gene expression related to reproductive status}

We quantified Gnrh mRNA expression in consecutive sections within the preoptic and anterior hypothalamus $(A C+4-A C-12)$. The total area covered by Gnrh mRNA that was analysed did not differ between reproductively active and suppressed females $(t=0.74$, $d f=14, P=0.47)$. Also, the average optical density was not different between the two groups $(t=0.52, d f=14$, $P=0.61)$. We further analysed the optical density and the area covered by the hybridization signal at different rostro-caudal levels. Measurement of the average optical density revealed no significant effect of status and brain region, but a significant interaction between both factors on Gnrh mRNA expression (status: $F_{1,14}=0.269, P=0.61$; region: $F_{16,224}=0.625$, $P=0.86$; interaction: $\left.F_{16,224}=2.21, P=0.006\right)$. Post hoc tests showed that breeding females had increased GNRH expression compared to non-breeding females in the anterior preoptic region $(A C+3$; Figs $1 A, G$ and 3), whereas the latter had elevated expression compared to breeders in the anterior hypothalamus at the level corresponding to the magnocellular division of the paraventricular nucleus (PALM) and the central portion of the supraoptic nucleus (SO, AC-10, AC-11, Figs 1C, I and 3). Measurement of the area size revealed no significant effect of status, but a significant effect of brain region and no significant interaction between both factors (status: $F_{1,14}=0.543$, $P=0.47$; region: $F_{16,224}=7.70, P<0.0001$; interaction: $\left.F_{16,224}=1.50, P=0.099\right)$.

\section{Discussion}

Here, we report for the first time the mRNA distribution and expression level of the GNRH gene in the mole-rat brain. In Damaraland mole-rats, female subordinates, in the presence of the queen, are physiologically suppressed to the extent of being anovulatory (Molteno \& Bennett 2000). We provide evidence for differential regulation of Gnrh mRNA in female breeders and non-breeders. Furthermore, the data suggest GNRH biosynthesis differs between neuron subpopulations.

\section{Distribution of Gnrh mRNA}

The distribution of Gnrh mRNA is in overall agreement with the previous description of the distribution of GNRH cell bodies detected by immunocytochemistry in the same species (Molteno et al. 2004). Similar to their findings, in the present study, the majority of labelling was found in rostro-caudal direction from the level of the medial septum up to the level of the suprachiasmatic nucleus $(\mathrm{SCH})$. In the tuberal hypothalamus, labelling was scarce and occurred close to the retrochiasmatic part of the supraoptic nucleus. Substantial species differences have been noticed in the distribution of GNRH neurons, with the opossum (Monodelphis domestica) having no GNRH neurons at all entering the hypothalamus, while two mustelid species, the mink (Neovison vison) and the ferret (Mustela putorius furo) constitute the other extreme with the major concentration of GNRH cell bodies in the mediobasal hypothalamus $(\mathrm{MBH}$, SchwanzelFukada et al. 1988, Toumi et al. 1992, Silverman et al. 1994). In most of the mole-rat species studied so far, the majority of cell bodies are found in the preoptic area (POA) and in the region of the medial septal nucleus (MS) and the diagonal band nucleus (NDB). Only few 
perikarya are localized in the $\mathrm{MBH}$ (Cape mole-rats, Natal mole-rats, Oosthuizen et al. 2008, Highveld molerats, Du Toit et al. 2006, naked mole-rats, Zhou et al. 2013, Damaraland mole-rats, Molteno et al. 2004). In contrast, in two species, the common mole-rat and Cape Dune mole-rat, about equal proportions of cell bodies are found in the MS/NDB/POA and in the $\mathrm{MBH}$ (Du Toit et al. 2006, Hart et al. 2008). This suggests that in the former species, the median eminence innervation is received mainly from neurons in the MS/NDB/ MPOA, whereas in the latter species, GNRH neurons located within the $\mathrm{MBH}$ could substantially contribute to the input into the median eminence. In the guinea pig, the small percentage of GNRH neurons within the arcuate nucleus that innervate the median eminence are those being essential for gonadotropin release (Silverman et al. 1994). The functional significance of the GNRH neuron subpopulations in the different mole-rat species remains to be determined.

\section{Influence of reproductive status on Gnrh mRNA levels}

In the present study, reproductive activity had no effect on the total area covered by Gnrh mRNA in the brain of female Damaraland mole-rats. This finding supports data from ICC studies in this species and other social molerats, which report similar total numbers of GNRH cell bodies and similar cell body size in breeder and nonbreeder females (Molteno et al. 2004, Du Toit et al. 2006, Oosthuizen et al. 2008, Zhou et al. 2013). However, our results show that reproductive status influences the regulation of the GNRH gene within specific neuron subpopulations. Our detailed analysis at different rostro-caudal levels of the brain identified significantly elevated Gnrh mRNA levels in breeders vs non-breeders in the anterior preoptic area, at one level of the MS/ $\mathrm{NDB}, \mathrm{AC}+3$. GNRH neurons of the MS/NDB/POA are implicated in the LH surge in female rats and hamsters as shown by double-label in situ hybridization for c-fos activation within GNRH neurons (Berriman et al. 1992, Wang et al. 1995). Furthermore, experiments involving electrical stimulation or deafferentation of the preoptic and anterior hypothalamic region strongly suggest that these neurons contribute to gonadotropin release and ovulation (Eskay et al. 1977, Palkovits et al. 1984, Köves \& Molnár 1986). However, only 50-70\% of the GNRH neurons actually innervate the median eminence, the remaining neurons project to other brain regions, and both types of neurons are intermingled (Silverman et al. 1987, Merchenthaler et al. 1989). This shows that the neurons regulating gonadotropin release are heterogeneously distributed, which makes it difficult to determine in the present study the functional significance of the observed mRNA expression at particular rostro-caudal levels. Four out of eight breeding Damaraland mole-rats were found to be in the stage of early to mid-pregnancy and for the others the exact stage of their estrous cycle at the time of sacrifice was unknown. The significantly elevated mRNA expression at the rostro-caudal level $\mathrm{AC}+3$ of these females despite their physiological differences suggests that the activity of the GNRH neurons in the anterior preoptic region is necessary for normal gonadotropin release. More detailed information could be obtained by analysing the Gnrh mRNA expression in breeder females according to their reproductive state. In rats, caudal to the suprachiasmatic nucleus few GNRH neurons are found that project to the median eminence and deafferentations in this region revealed that only about $10 \%$ of the GNRH content of the median eminence originates from these neurons (Köves \& Molnár 1986, Merchenthaler et al. 1989). This may also apply to Damaraland mole-rats as the GNRH neuron distribution is largely similar to that in rats. Therefore, the increased Gnrh mRNA expression in this region $(A C+10, A C+11)$ of subordinate females is likely to concern GNRH neurons that have other functions in the brain than regulation of gonadotropin release.

With our method of in situ hybridization, we cannot distinguish whether the increase in Gnrh mRNA that we observed at different rostro-caudal levels in the brain of breeding and non-breeding females was due to transcriptional or post-transcriptional regulation of the gene, the latter occurring through increased stability of the primary transcript (Gore \& Roberts 1995). However, in female rats in proestrus before the LH surge, the GNRH gene is transcriptionally activated as the rise in cytoplasmatic mRNA levels is preceded by a rise in primary transcript (Gore \& Roberts 1995). Further, oestradiol treatment of female rats increases the GNRH gene transcription rates in the region of the organum vasculosum of the lamina terminalis (OVLT)/ rostral preoptic area before the LH surge, suggesting that transcriptional regulation of the GNRH gene is involved in ovulation (Petersen et al. 1996). ERo-expressing neurons residing within the anteroventral periventricular nucleus (AVPV) are responsible for the oestrogen-positive feedback on GNRH neurons, which is essential for the LH surge (Wintermantel et al. 2006). These neurons co-express kisspeptin (Kiss 1), which is a potent activator of GNRH release and oestradiol upregulates Kiss 1 mRNA expression in this area (for review, see Smith 2013). We have recently shown that breeding female Damaraland mole-rats exhibit significantly higher ERo-expression within the anteroventral periventricular nucleus than non-reproductives (Voigt et al. 2014). Therefore, reduced availability of oestradiol in reproductively suppressed females in this region could lead, due to insufficient Kiss 1 expression, to reduced levels of GNRH gene transcription and ultimately to the observed anovulatory phenotype. Further experimental work is required to elucidate the kisspeptin-GNRH pathway and to determine its role in regulating reproductive activity in a mole-rat species with socially induced infertility. 


\section{Declaration of interest}

The authors declare that there is no conflict of interest that could be perceived as prejudicing the impartiality of the research reported.

\section{Funding}

This work was supported by a fellowship from the University of Pretoria to $\mathrm{CV}$. We are grateful to the DST-NRF for funding to $\mathrm{N} \mathrm{B}$.

\section{Acknowledgements}

Permits were obtained from the Northern Cape Department of Nature Conservation. We thank the Family Reynecke for allowing us to work on their farm. We are grateful to Manfred Gahr and Stefan Leitner for support and Carolina FranklVilches and Moritz Hertel of the Department of Behavioural Neurobiology, Max Planck Institute of Ornithology for help in cloning the mole-rat fragment.

\section{References}

Abbott DH 1984 Behavioral and physiological suppression of fertility in subordinate marmoset monkeys. American Journal of Primatology 6 169-186. (doi:10.1002/ajp.1350060305)

Bennett NC, Jarvis JUM, Faulkes CG \& Millar RP 1993 LH responses to single doses of exogenous $\mathrm{GnRH}$ by freshly captured Damaraland molerats, Cryptomys damarensis. Journal of Reproduction and Fertility 99 81-86. (doi:10.1530/jrf.0.0990081)

Bennett NC 1994 Reproductive suppression in social Cryptomys damarensis colonies: a lifetime of socially-induced sterility in males and females. Journal of Zoology 234 25-39. (doi:10.1111/j.1469-7998.1994. tb06054.x)

Bennett NC, Jarvis JUM, Millar RP, Sasano H, \& Ntshinga KV 1994 Reproductive suppression in eusocial Cryptomys damarensis colonies: socially-induced infertility in females. Journal of Zoology 233 617-630. (doi:10.1111/j.1469-7998.1994.tb05369.x)

Berriman SJ, Wade GN \& Blaustein JD 1992 Expression of Fos-like proteins in gonadotropin-releasing hormone neurons of Syrian hamsters: effects of estrous cycles and metabolic fuels. Endocrinology 131 2222-2228. (doi:10.1210/endo.131.5.1425420)

Clayton RN 1982 Gonadotropin-releasing hormone modulation of its own pituitary receptors: evidence for biphasic regulation. Endocrinology 111 152-161. (doi:10.1210/endo-111-1-152)

Du Toit L, Bennett NC, Katz AA, Kalló I \& Coen CW 2006 Relations between social status and the gonadotrophin-releasing hormone system in females of two cooperatively breeding species of African mole-rats, Cryptomys hottentotus hottentotus and Cryptomys hottentotus pretoriae: neuroanatomical and neuroendocrinological studies. Journal of Comparative Neurology 494 303-313. (doi:10.1002/ cne.20792)

Eskay RL, Mical RS \& Porter JC 1977 Relationship between luteinizing hormone releasing hormone concentration in hypophysial portal blood and luteinizing hormone release in intact, castrated, and electrochemically-stimulated rats. Endocrinology 100 263-270. (doi:10.1210/endo-100-2-263)

Faulkes CG \& Bennett NC 2001 Family values: group dynamics and social control of reproduction in African mole-rats. Trends in Ecology and Evolution 16 184-190. (doi:10.1016/S0169-5347(01)02116-4)

Faulkes CG, Abbott DH \& Jarvis JU 1990 Social suppression of ovarian cyclicity in captive and wild colonies of naked mole-rats, Heterocephalus glaber. Journal of Reproduction and Fertility 88 559-568. (doi:10.1530/ jrf.0.0880559)
Gahr M \& Metzdorf R 1997 Distribution and dynamics in the expression of androgen and estrogen receptors in vocal control systems of songbirds. Brain Research Bulletin 44 509-517. (doi:10.1016/S03619230(97)00233-5)

Gore AC \& Roberts JL 1995 Regulation of gonadotropin-releasing hormone gene expression in the rat during the luteinizing hormone surge. Endocrinology 136 889-896. (doi:10.1210/en.136.3.889)

Hart L, Bennett NC, Kalamatianos T, Oosthuizen MK, Jarvis JU, O'Riain MJ \& Coen CW 2008 Neuroanatomical investigation of the gonadotrophin-releasing hormone 1 system in the seasonally breeding Cape dune mole-rat, Bathyergus suillus. Brain Research Bulletin 77 185-188. (doi:10.1016/j.brainresbull.2008.07.004)

Jimenez-Linan M \& Rubin BS 2001 Dynamic changes in luteinizing hormone releasing hormone transcriptional activity are associated with the steroid-induced LH surge. Brain Research 922 71-79. (doi:10.1016/ s0006-8993(01)03151-1)

Jimenez-Linan M, Rubin BS \& King JC 1997 Examination of guinea pig luteinizing hormone-releasing hormone gene reveals a unique decapeptide and existence of two transcripts in the brain. Endocrinology 138 4123-4130. (doi:10.1210/en.138.10.4123)

Kalamatianos T, du Toit L, Hrabovszky E, Kallo I, Marsh PJ, Bennett NC \& Coen CW 2005 Characterization of gonadotrophin-releasing hormone precursor cDNA in the Old World mole-rat Cryptomys hottentotus pretoriae: high degree of identity with the New World guinea pig sequence. Journal of Neuroendocrinology 17 265-268. (doi:10.1111/ j.1365-2826.2005.01307.x)

Keller L \& Reeve KH 1994 Partitioning of reproduction in animal societies. Trends in Ecology and Evolution 9 98-102. (doi:10.1016/01695347(94)90204-6)

Köves K \& Molnár J 1986 Effect of various hypothalamic deafferentations injuring different parts of the GnRH pathway on ovulation, GnRH content of the median eminence, and plasma LH and FSH levels. Neuroendocrinology 44 172-183. (doi:10.1159/000124642)

Merchenthaler I, Setalo G, Csontos C, Petrusz P, Flerko B \& Negro-Vilar A 1989 Combined retrograde tracing and immunocytochemical identification of luteinizing hormone-releasing hormone- and somatostatin-containing neurons projecting to the median eminence of the rat. Endocrinology 125 2812-2821. (doi:10.1210/endo-125-6-2812)

Molteno AJ \& Bennett NC 2000 Anovulation in non-reproductive female Damaraland mole-rats (Cryptomys damarensis). Journal of Reproduction and Fertility 119 35-41. (doi:10.1530/jrf.0.1190035)

Molteno AJ, Kallo I, Bennett NC, King JA \& Coen CW 2004 A neuroanatomical and neuroendocrinological study into the relationship between social status and GnRH system in cooperatively breeding female Damaraland mole-rats, Cryptomys damarensis. Reproduction 127 13-21. (doi:10.1530/rep.1.00048)

Oosthuizen MK, Bennett NC \& Coen CW 2008 An immunohistochemical study of the gonadotrophin-releasing hormone 1 system in solitary Cape mole-rats, Georychus capensis, and social Natal mole-rats, Cryptomys hottentotus natalensis. Neuroscience 157 164-173. (doi:10.1016/j. neuroscience.2008.08.054)

Palkovits M, Pattou E, Herman JP \& Kordon C 1984 Mapping of $\mathrm{LH}-\mathrm{RH}$-containing projections to the mediobasal hypothalamus by differential deafferentation experiments. Brain Research 298 283-288. (doi:10.1016/0006-8993(84)91427-6)

Paxinos G \& Watson C 2007 The Rat Brain in Stereotaxic Coordinates. Amsterdam, Netherlands: Academic Press.

Petersen SL, Gardner E, Adelman J \& McCrone S 1996 Examination of steroid-induced changes in LHRH gene transcription using 33P-and 35S-labeled probes specific for intron 2. Endocrinology 137 234-239. (doi:10.1210/endo.137.1.8536618)

Schwanzel-Fukada M, Fadem BH, Garcia MS \& Pfaff DW 1988 Immunocytochemical localization of luteinizing hormone-releasing hormone (LHRH) in the brain and nervus terminalis of the adult and early neonatal gray short-tailed opossum (Monodelphis domestica). Journal of Comparative Neurology 276 44-60. (doi:10.1002/ cne.902760104)

Silverman AJ, Jhamandas J \& Renaud LP 1987 Localization of luteinizing hormone-releasing hormone $(\mathrm{LHRH})$ neurons that project to the median eminence. Journal of Neuroscience 7 2312-2319.

Silverman AJ, Livne I \& Witkin JW 1994 The gonadtropin-releasing hormone, neuronal systems: immunocytochemistry and in situ 
hybridization. In The Physiology of Reproduction, edn 2, pp 1683-1709. Eds E Knobil \& JD Neill. New York, NY, USA: Raven Press.

Smith JT 2013 Sex steroid regulation of kisspeptin circuits. Advances in Experimental Medicine and Biology 784 275-295. (doi:10.1007/978-14614-6199-9_13)

Snyman PC, Jackson CR \& Bennett NC 2006 Do dispersing female Damaraland mole-rats, Cryptomys damarensis (Rodentia: Bathyergidae) exhibit spontaneous or induced ovulation? Physiology and Behavior $\mathbf{8 7}$ 88-94. (doi:10.1016/j.physbeh.2005.09.003)

Spratt DP \& Herbison AE 1997 Regulation of preoptic area gonadotrophinreleasing hormone $(\mathrm{GnRH}) \mathrm{mRNA}$ expression by gonadal steroids in the long-term gonadectomized male rat. Brain Research: Molecular Brain Research 47 125-133. (doi:10.1016/S0169-328X(97)00037-5)

Swanson LW 2004 Brain Maps: Structure of the Rat Brain. Amsterdam, Netherlands: Academic Press.

Toumi FN, Martinet L \& Peytevin J 1992 Gonadotropin-releasing hormone neurons and pathways in the brain of the female mink (Mustela vison). Cell and Tissue Research 270 383-393. (doi:10.1007/ BF00328022)

Voigt C, Gahr M, Leitner S, Lutermann H \& Bennett NC 2014 Breeding status and social environment differentially affect the expression of sex steroid receptor and aromatase mRNA in the brain of female Damaraland mole-rats. Frontiers in Zoology 1138. (doi:10.1186/1742-9994-11-38)

Wang HJHoffman GE \& Smith MS, 1995 Increased GnRH mRNA in the $\mathrm{GnRH}$ neurons expressing cFos during the proestrous LH surge. Endocrinology 136 3673-3676. (doi:10.1210/en.136.8.3673)

Whitfield HJ Jr, Brady LS, Smith MA, Mamalaki E, Fox RJ \& Herkenham M 1990 Optimization of CRNA probe in situ hybridization methodology for localization of glucocorticoid receptor mRNA in rat brain: a detailed protocol. Cellular and Molecular Neurobiology 10 145-157. (doi:10.1007/BF00733641)

Wintermantel TM, Campbell RE, Porteous R, Bock D, Gröne HJ, Todman MG, Korach KS, Greiner E, Pérez CA, Schütz G et al. 2006 Definition of estrogen receptor pathway critical for estrogen positive feedback to gonadotropin-releasing hormone neurons and fertility. Neuron $\mathbf{5 2}$ 271-280. (doi:10.1016/j.neuron.2006.07.023)

Wise PM, Rance N, Selmanoff M \& Barraclough CA 1981 Changes in radioimmunoassayable luteinizing hormone-releasing hormone in discrete brain areas of the rat at various times on proestrus, diestrous day 1, and after phenobarbital administration. Endocrinology 108 2179-2185. (doi:10.1210/endo-108-6-2179)

Zhou S, Holmes MM, Forger NG, Goldman BD, Lovern MB, Caraty A, Kalló I, Faulkes CG \& Coen CW 2013 Socially regulated reproductive development: analysis of $\mathrm{GnRH}-1$ and kisspeptin neuronal systems in cooperatively breeding naked mole-rats (Heterocephalus glaber). Journal of Comparative Neurology 521 3003-3029. (doi:10.1002/cne.23327)

Zoeller RT \& Young WS 3rd 1988 Changes in cellular levels of messenger ribonucleic acid encoding gonadotropin-releasing hormone in the anterior hypothalamus of female rats during the estrous cycle. Endocrinology 123 1688-1689. (doi:10.1210/endo-123-3-1688)

Received 23 August 2016

First decision 26 September 2016

Revised manuscript received 8 January 2017

Accepted 19 January 2017 